\title{
Optical Control of Resonance Energy Transfer in Quantum Dot Systems
}

\author{
Dilusha Weeraddana ${ }^{1}$, Malin Premaratne ${ }^{1}$ and David L. Andrews ${ }^{2}$
}

\begin{abstract}
We demonstrate that the rate of resonance energy transfer can be extensively controlled through an applied offresonant radiation field under favourable physical configurations of the quantum dots.
\end{abstract}

\section{INTRODUCTION}

Nanotechnology offers means to study and fabricate atomlike nanostructures with discrete energy transitions, which arise from band splitting due to the quantum confinement effect, commonly termed quantum dots (QDs). Thus, quantum dots are becoming increasingly prominent at the very heart of modern nanophotonics [1], [2]. Moreover, the excitonexciton interactions through nonradiative resonance energy transfer (RET) in QDs has enabled exciting opportunities in many applications. It emerges that a throughput off-resonant laser beam can produce significant additional effects on the process of RET between two nanoparticles [5]. This is due to coupled absorption and stimulated emission of photons from and into the applied beam. Nevertheless, a complete understanding of laser controlled RET in dimensionality constrained nanostructures (i.e. quantum dots) is still lacking. The origin of these complications is deeply rooted in the nature of the exciton and photons mediating the transfer process. Therefore, In this paper, our main aim is to study the effects on the RET process due directly to a laser field impinging on a QD system.

\section{Optically CONTROLLED RET}

Since the RET process is fully quantum mechanical in nature, it can be formally described within the framework of quantum electrodynamics (QED) [3], [4]. We first derived the optically controlled RET rate for QDs by using the second and fourth order time dependent perturbation theory, and.by applying the Fermi's golden rule [3], $\Gamma^{\text {laser }} \overline{\overline{=}}$ $2 \pi \rho . \quad(2) .+2 \pi \rho \cdot M(4)+4+4 \rho \operatorname{Re}\left\{M(2) M^{(4)}\right\}$ where $\mathrm{k}$ is the reduced Plarík's constant, $\beta$ is the density of final states of the acceptor, $M^{(2)}, M^{(4)}$ are second and fourth order coupling matrix elements respectively. Furthermore, the third term represents the quantum interference of the RET process and signifies the controllability of the RET due to the laser field. Here, the off-resonant laser field is applied to stimulate and control the energy transfer process [5], [3] (see Fig. 1 (a)).

${ }^{1}$ Dilusha Weeraddana, Malin Premaratne are with Advanced Computing and Simulation Laboratory $(\mathrm{A} X \mathrm{~L})$, Department of Electrical and Computer Systems Engineering, Monash University, Clayton, Victoria, 3800, Australia dilusha.weeraddana@monash.edu, malin.premaratne@monash.edu

${ }^{2}$ David L. Andrews with School of Chemistry, University of East Anglia, Norwich Research Park, Norwich NR4 7TJ, United Kingdom d. l. andrews@uea.ac.uk

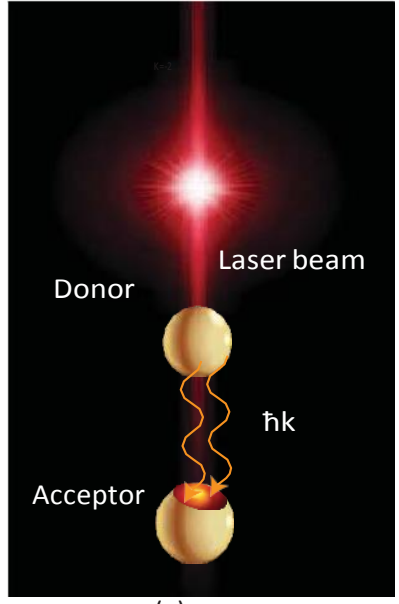

(a)

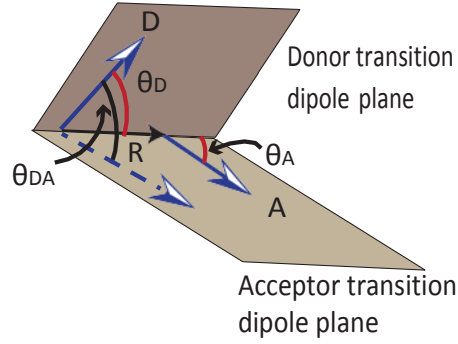

(b)
Fig. 1. Schematics for the (a) laser assisted resonance energy transfer; (b) relative angles associated with the quantum dots transition dipole moments, $R$ is the distance between two quantum dots.

\section{DESIGN GUIDELINES}

a) RET rate enhancement: The RET rate can be enhanced efficiently, when the optimal configuration of the QD particles is implemented. Here, fast transfer occurs between nearby sites when the QD transition dipole moments and the separation vectors are collinear $\left(\theta_{D A}=\theta_{D}=\theta_{A}=0\right.$, corresponding angles are given in Fig. 1 (b)). The transfer rate enhancement with increasing laser intensity for this scenario is depicted in Fig. 2(a), (d). The results demonstrate that even for moderate laser intensities $\left(I \approx 10^{16} \mathrm{Wm}^{-2}\right)$, up to $50 \%$ rate enhancements can be achieved.

b) RET rate deterioration: When $\cos \left(\theta_{D A}\right)=\pi$, $\cos \left(\theta_{D}\right)=\pi$. (or 0$)$ and $\cos \left(\theta_{A}\right)=0($ or $\pi)$, total energy transfer efficiency declines until it reaches its minimum

point when $/$ becomes $2.66 \times 10^{17} \mathrm{Wm}^{-2}$. After this point, the total energy transfer rate steadily elevates its value and finally starts dominating the direct transfer rate when I “ $5.32 \times 10^{17} \mathrm{Wm}^{-2}$ (see point $X$ in Fig. 2 (b)). Fig. 2 (e) illustrates reduction and enhancement of the transfer rate as a function of increasing laser intensity.

c) All-optical switching: The direct coupling has been "switched off" between QD particles by arranging them such that the transition dipole moments are perpendicular to each other and donor-acceptor separation vector, allowing full control to the laser driven transfer (see Fig. 2 (c)). In this case, optical switching can be produced by the throughput of a single off-resonant beam [6], [7].

d) Distance dependence: Owing to the $(R)^{-6}$ distance dependence on the total transfer rate [3], lower relative 


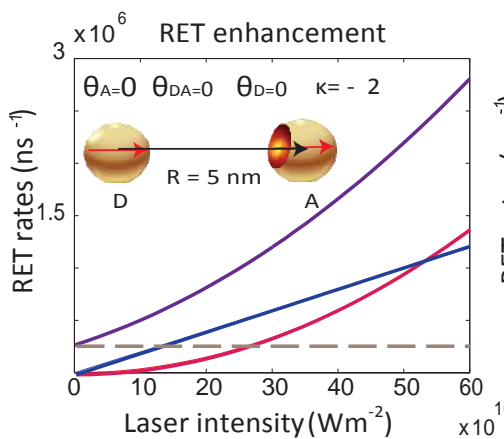

(a)

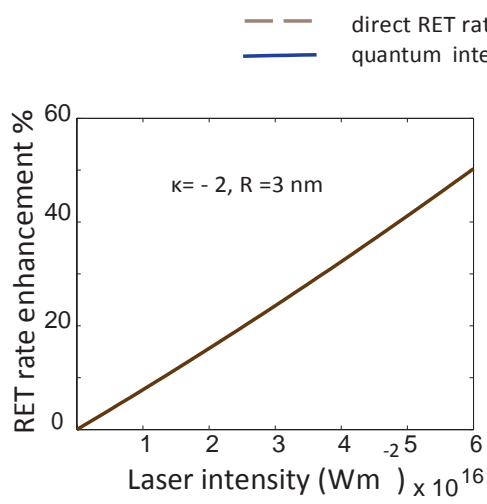

(d)

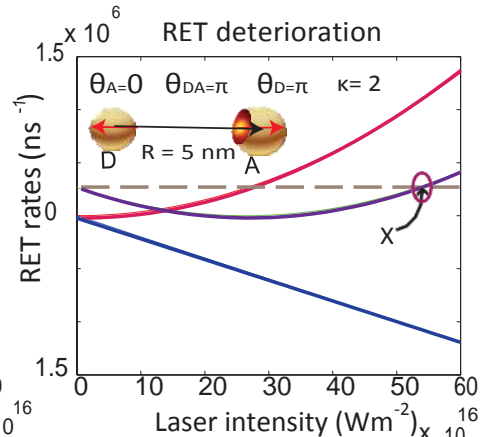

(b)

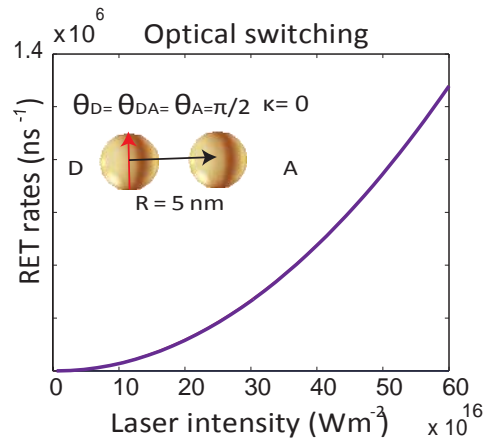

(c)

$-2 \leq \kappa \leq 2$, is the orientation factor

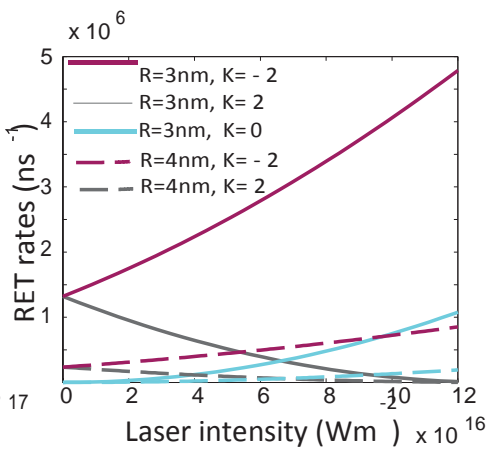

(f)

Fig. 2. Transfer rates of the direct RET, laser driven RET, quantum interference and the total RET rate for three non-trivial geometries $(\kappa=-2,2,0)$ are shown in (a)-(c) respectively as a function of the laser intensity; (d) percentage of rate enhancement when $K=-2$; (e) percentage of rate enhancement and deterioration when $K=2$; (f) transfer rate as a function of $I$, for three orientational factors $(\kappa=-2,2,0)$ and corresponding two relative distances ( $3 \mathrm{~nm}, 4 \mathrm{~nm}$ ) between $D$ and $A$. The solid and dashed lines represent rates for $3 \mathrm{~nm}$ and $4 \mathrm{~nm}$ respectively.

spacing between donor and acceptor particles leads to a higher transfer efficiency as shown in Fig. 2 (f).

It can be seen from the results that the RET efficiency can be effectively controlled by altering the laser irradiance, orientation of transition dipoles and also by varying the separation distance of QD pair. It is also important that the laser radiation should be off-resonant, in order to prevent direct excitation of either QD.

\section{CONCLUSION}

In this paper, we have demonstrated the possibility of altering the strength and directivity of RET between two QDs by careful engineering of the geometry of the system and applying an auxiliary laser beam, hence altering the efficiency of the RET. Further, we have proposed design guidelines for controlling RET, using quantum electrodynamical tools. In addition, the proposed design guidelines can help in the optimization and the design of novel antenna devices for high efficiency light harvesting systems and hold potential for optical transistors, optical logic gates.

\section{REFERENCES}

[1] I. L. Medintz, H. Mattoussi, "Quantum dot-based resonance energy transfer and its growing application in biology," Semiconductor Science and Technology, vol. 11, no. 1, pp. 17-45, 2009.
[2] B. Dong, C. G. Bailey, A. Afanasev and M. Zaghloul, "Modeling and simulation of InAs/GaAs quantum dot solar cells in SILVACO TCAD," in Photovoltaic Specialist Conference (PVSC), 2014 IEEE 40th, pp. 0476-0478, IEEE, 2014.

[3] D. P. Craig and T. Thirunamachandran, Molecular Quantum Electrodynamics. Dover, New York, 1998.

[4] D. Weeraddana, M. Premaratne, and D. L. Andrews, "Direct and thirdbody mediated resonance energy transfer in dimensionally constrained nanostructures," Physical Review B, vol. 92, no. 3, p. 035128, 2015.

[5] P. Allcock, R. D. Jenkins, and D. L. Andrews, "Laser-assisted resonance-energy transfer," Physical Review A, vol. 61, no. 2, p. 023812 , 2000.

[6] D. S. Bradshaw and D. L. Andrews, "Optical control and switching of excitation transfer in nano-arrays," in Lasers and Applications in Science and Engineering, pp. 687500-68750O, International Society for Optics and Photonics, 2008.

[7] M. Bates and T. R. Blosser, X. Zhuang "Short-range spectroscopic ruler based on a single-molecule optical switch," Phys. Rev. Lett., vol. 94, no. 10, p. 108101, 2005. 